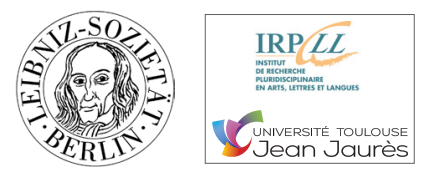

\title{
Die Herausgeberinnen im Gespräch
}

\section{MIT WELCHEN FORSCHUNGSPROJEKTEN BESCHÄFTIGEN SIE SICH GEGENWÄRTIG?}

\section{DR:}

Kürzlich ist meine kulturwissenschaftliche Studie zur Vita der international bekannten Zola-Forscherin Rita Schober erschienen (Narr 2018). Mit dieser Arbeit sollen Potenzen kulturwissenschaftlicher Forschung für die (auto)biographische Fachgeschichtsschreibung verdeutlicht werden. „Das Fremde als Anspruch“ ist der Titel einer kulturtheoretischen Studie, die ebenfalls gerade abgeschlossen ist und in einen Band zum Thema "Interkulturalität und Migration" integriert ist. Phänomenologische und systemtheoretische Ansätze werden dabei auf ihre Eignung zur wissenschaftlichen Bearbeitung von Fremderfahrungen geprüft.

Des Weiteren arbeite ich seit vielen Jahren an einem größeren Forschungsfeld: Bildung vs formation de la raison? Dabei geht es um die Frage nach Gemeinsamkeiten und Unterschieden in den Konzepten und Praktiken des Lehrens, Lernens und Studierens in deutschen und französischen Bildungsinstitutionen. Eine der zentralen Fragen dieser vergleichenden Forschung lautet, wie sich bestimmte Ideen aus der vielschichtigen Ideengeschichte an Praxen binden können und in bestimmten sozialen Kontexten in einen Habitualisierungsprozess einmünden und damit längerfristig mentalitätsbildend für bestimmte Gruppen wirken. Ideengeschichte wird auf diese Weise mit der sozialen Praxis verbunden. Die vergleichende Perspektive wird durch eine interkulturelle ergänzt. Diese fungiert als wesentliche Heuristik, indem auch empirische Befunde zu Missverständnissen und Hürden beim Studieren im Nachbarland thematisiert werden. „Studieren in Frankreich und Deutschland“ (2006 dt., 2007 frz.) sowie „Formation de la raison“ (2012) bilden die Grundlage für weitere Einzelstudien zu diesem kulturellen Muster, die derzeit der Julimonarchie in Frankreich gewidmet sind.

\section{FK:}

Teufel und Gespenster: Glauben und literarisches Spie / ist der Titel der vorletzten von mir als Co-Herausgeberin veröffentlichten Forschungsarbeit, die sich mit dem Ziel verband, zu zeigen wie sich Positionen der Eliten und die Volkspraktiken in der Frage von Aberglauben und seiner Bekämpfung durch die geistigen Eliten verschränken und durch eine Art poröse Beziehung gekennzeichnet werden können. Diese KollektivArbeit, die unlängst veröffentlich wurde, widmet sich diesen Beziehungen vor allem unter dem Aspekt des epistemologischen Umbruchs um 1800, der ideologische, politische, kulturelle und ästhetische Folgen hatte und ermöglichte, dass sich der moderne literarische Diskurs nicht nur über Teufel und Gespenster amüsiert, sondern auch nach der Schuld und Verantwortung des modernen Menschen für das Böse fragt, das nunmehr säkular aufgefasst wird. Ein dann folgender Sammelband beschäftigt sich mit „Briefkunst zwischen Höflichkeit und staatsbürgerlichem Pflichtgefühl“ von Gellert bis Grass. Dabei wird die Entwicklung einer Aufwertung des Briefschreibens in der Zeitspanne vom 18. bis zum 20. Jh. untersucht, in einem Moment, wo sich die deutsche Briefkultur von dem französischen Modell emanzipiert. Der Briefschreiber wird in dieser Konzeption des Buches entsprechend seiner Eingliederung in eine Gemeinschaft erfasst, sei diese freiwillig, per Zwang oder durch eine affektive und freie Beziehung zur Gemeinschaft hergestellt. Die Briefform der Höflichkeit errang eine soziale und philosophische Dimension, trug den Veränderungen Rechnung, die sich mit den anthropologischen Paradigmenwechseln zu Beginn des 19. Jh. verbanden, indem sie eine Ausdifferenzierung der Brieftypologie simulierte, nämlich eine Verringerung der rhetorischen Verwendungsweisen zugunsten eines politischen und sozialen Engagements.

Eine meiner aktuellen Beschäftigungen schreibt sich in diese Überlegungen ein: der Briefwechsel des Erzherzogs Johann von Österreich, der in weiten Teilen unveröffentlicht geblieben ist. Seine Überlegungen über „das Volk der Steiermark“ lassen uns viel über die Vorstellungen dieses Erzherzogs, den Pionier der „Volkskunde“, über die staatsbürgerliche Erziehung erfahren. Ein anderes Projekt betrifft eine kommentierte Edition der Reiseberichte von Johann Georg Sulzer, ein Band, der als Teil einer Gesamtausgabe seiner Werke (im Verlag Schwabe) geplant ist. Und schließlich möchte ich weiter über die Reiseschriftsteller schreiben, über ihre Begegnung mit fremden Gemeinschaften, ihre Alteritätserfahrungen. Sie sind Träger von Normen ihrer Herkunftskulturen, insbesondere in philosophischer und religiöser Hinsicht, dies macht sie besonders sensibel für Integrations- bzw. Exklusionsprozesse in den von ihnen besuchten Gesellschaften und Kulturen. 
MTM:

Ich habe 2017 meine Promotion abgeschlossen mit einer Arbeit zur Wissenskultur der französischen Eliten am Ende des 18. Jahrhunderts (Königshausen \& Neumann 2019). Die Arbeit war als Teilprojekt des von Dorothee Röseberg genannten kulturellen Musters formation de la raison angelegt. Dabei handelt es sich um ein Muster mit dem zentralen Begriffskonzept des raisonnement, das bestimmten gedanklichen Operationen Priorität gibt. Anders als die bisherige Forschung belegt, hat sich ein solches Muster bereits nachweislich über die Philosophie des sensualistischen Rationalismus herausgebildet und konnte sich nach der Revolution in eine für die französischen Eliten konzipierten Bildungspraxis mit nationalem und modellhaftem Anspruch etablieren. Meine Untersuchungen beziehen sich auf die institutionellen Netzwerke der Idéologues, wo ich im Ergebnis zeigen konnte, welchen wesentlichen Anteil die Idéologues an der Verstetigung des kulturellen Musters haben. Erste Ergebnisse habe ich in dem gemeinsam mit Jenny Ettrich herausgegebenen Tagungsband Dialogpotenziale kulturwissenschaftlicher Forschung in den Fremdsprachenphilologien (Peter Lang 2018) präsentiert. Derzeit bin ich Postdoktorandin an der Universität Bremen und koordiniere das DFG-Verbund-Projekt „Transatlantische Ideenzirkulation und -transformation: Die Wirkung der Aufklärung in den neueren frankokaribischen Literaturen“. Hier beschäftige ich mich aus einer kulturwissenschaftlichen und transnationalen Perspektive mit den Zirkulationsprozessen, die das Wissen und die Aushandlungen über die Natur in frankokaribischen Texten vom 18. Jahrhundert bis heute einnehmen.

\section{$\mathrm{CM}$ :}

Ein erstes großes Forschungsensemble, mit dem ich mich seit vielen Jahren beschäftige, erstreckt sich auf die internationalen Filmfestivals in Europa während des Kalten Krieges. Den Ausgangspunkt meiner Forschung bildete die Studie über das Internationale Leipziger Festival für Dokumentar- und Animationsfilm in der DDR, welches 1955 gegründet wurde und seither jährlich stattfindet (Cinéma et guerre froide, Paris, 2014 und die engl. Übersetzung, Screened Encounters, New York, 2018). Ich untersuche die Festivalveranstaltungen hinsichtlich der internationalen und transnationalen Zirkulationslogiken, die, ungeachtet der diplomatischen Spaltungen, zwischen Ost und West, aber auch zwischen Nord und Süd, vorhanden waren. Der Schwerpunkt der Analyse liegt nicht nur auf den Institutionen, sondern auch auf den verschiedenen persönlichen Werdegängen und den Filmen, also auf zentralen Elementen des Austausches. Entlang dieser und weiterer Fragen habe ich gemeinsam mit Kollegen aus Frankreich, Deutschland und Italien ein Forschungsprojekt ins Leben gerufen, das die transnationalen Verflechtungen der europäischen und außereuropäischen Filmschulen in den Blick nimmt. Zu einem weiteren Forschungsfeld gehören meine jüngsten Studien zur Geschichte der internationalen Solidaritätsbewegungen mit Chile in Europa nach dem Putsch vom 11. September 1973 und der anschließenden Machtergreifung des Militärs. Dabei widmet sich einer meiner Forschungszweige der Laufbahn und dem Engagement des westdeutschen Pastors Helmut Frenz (1933-2011), einer Schlüsselfigur der Solidarität zwischen Lateinamerika und Europa.

\section{WIE SIND SIE ZUR HISTOIRE CULTURELLE I ZU DEN ÉTUDES CULTURELLES / ZUR KUL- TURWISSENSCHAFT GEKOMMEN?}

\section{DR:}

Ich könnte hier eine lange Geschichte von Hindernissen der wissenschaftlichen Profilierung erzählen, denn in der Romanistik als Geisteswissenschaft und Fremdsprachenphilologie gab es nach 1945 bis zu den 1990er Jahren keine Kulturwissenschaft und sie ist dort auch heute keineswegs überall etabliert. Dieser Befund gilt vor allem, wenn man Kulturwissenschaft als Brücke zwischen Geistes- und Sozialwissenschaften versteht, wie dies in meinen Arbeiten der Fall ist. Begonnen hat alles an der Humboldt-Universität, wo ich während meiner wissenschaftlichen Ausbildung von Kooperationen mit Historikern und den Arbeiten des Instituts für Kulturwissenschaft profitieren konnte. Trotz der bis 1989 ideologisch und material begrenzten Forschungsmöglichkeiten in der DDR lässt sich im Rückblick eine kontinuierliche Arbeit ,zwischen den Stühlen', an den Rändern etablierter Disziplinen bzw. im Überschreiten ihrer Grenzen erkennen. Daraus ergeben sich über längere Zeit die Randpositionen meiner Arbeiten in den Publikationsorganen sehr unterschiedlicher Disziplinen. Von Historikern begleitet widmete sich meine Diplomarbeit einer Medienanalyse und befasste sich mit dem diskursiven Feld im Kontext der Machtübernahme Charles de Gaulles 1958. Es folgte eine Periode wissenschaftlicher Assistenz, in der meine Arbeiten Teil größerer literaturwissenschaftlicher Projekte waren, aber dort eine gewisse Exotik darstellten: Studien zur Geschichte der Funktionen von Literatur für die französischen Eliten, zur Geschichte des Literatur-Kanons in Frankreich und schließlich zu einer vergleichenden empirischen Leseforschung. Mit meiner Berufung auf einen Lehrstuhl für Landes- und Kulturwissenschaft romanischer Länder beginnt eine zweite Periode, die thematisch mit einer ,Emanzipation" von der Literaturwissenschaft verbunden und stärker auf eine eigenständige theoretische und methodologische Profilierung von Kulturwissenschaft in den Fremdsprachenphilologien gerichtet ist. Besonders wichtig ist mir dabei die Thematisierung des Blickes, mit dem ein Gegenstand untersucht wird. Konzepte zum Erfassen verschiedener Formen des Kulturkontaktes und ihrer ,Ergebnisse;, ob in Medien oder in situativen Begegnungen untersucht, sowie das Problem der Fremdheit bzw. Fremderfahrung stehen epistemologisch im Zentrum meiner Forschungen. Mein Kulturverständnis war stets nicht normativ, auch auf Praxen 
orientiert und hat sich dabei in eine sozio-anthropologische Richtung entwickelt. Zugleich bin ich in dem Sinne Philologin geblieben, als die Untersuchung der sprachlichen Kommunikationsformen für mich bei allen Untersuchungen unverzichtbar ist.

\section{FK:}

Für eine französische Germanistin konnte der Weg dahin letztlich kohärent verlaufen. Zu Beginn ging es im Rahmen meiner Forschungen zu den deutsch-französischen Reiseberichten des 18. Jh. darum, eine wissenschaftliche Legitimation für die Spezifik der entsprechenden Diskurse zu finden, die damals, aufgrund ihrer Hybridität, noch nicht zu akademischen Würden gelangt waren. Hinzu kam eine andere Besonderheit, die sich mit der Entwicklung und Anerkennung dieses Diskurses in einer bestimmten Generation verband: Selbst wenn die Reisenden nach 1800 zu einer Traditionsbildung beitrugen, in der in Diskursen geographische, ökonomische und soziologische Gegebenheiten der bereisten Gebiete vermittelt wurden, so veränderten sich die Wahrnehmungsweisen periodisch. Diejenigen des 19. Jh. implizierten mehr und mehr das kulturelle Leben des betreffenden Volkes, eine Berücksichtigung der Landschaften und den Ausdruck von religiöser Sensibilität. Dies alles führte zu einer Anerkennung dieser Diskurse als Untersuchungsobjekt. Der Begriff und das Konzept der Mentalitätsgeschichte, die damals vorherrschend waren, als ich meine Forschungen unternahm, schienen dafür adäquat zu sein. Es ist für mich aber evident, dass die Begriffskonzepte der histoire culturelle für das Genre der Reisebeschreibungen adäquater sind, weil sie nützlich sein können, um die Qualität der Diskurse zu bestimmen. Gleichzeitig kann auf die Frage eingegangen werden, wie Autor innen ihre Identität ins Spiel bringen, welcher Grad an Repräsentativität dabei jeweils vorliegt und wie sich die kulturelle Prägung der Autor_innen letztlich in die Diskurse einschreibt. Seit Anfang der 1990er Jahre konnte ich als Lehrende von der institutionellen Anerkennung der civilisation profitieren. Ich schlug Studierenden und Doktorand innen Themen vor, die sich am Schnittpunkt von Literatur- und Geschichtswissenschaft verorteten. Die textuellen Träger einer solchen Scharnierfunktion waren dabei zentral. Um ein jüngstes Beispiel zu nennen: Untersuchungen journalistischer Feuilletons der Jahre 1914-18 erlaubten es, die delikate Frage einer „Kriegskultur“ zu erörtern und die Kreativität und Modernität einiger Autoren zu studieren, wobei Literatur und Geschichte nicht voneinander zu trennen waren.

\section{MTM:}

Mein Weg zur Kulturwissenschaft steht in einem engen Zusammenhang mit meinem Studium der Frankoromanistik an der Martin-Luther-Universität Halle-Wittenberg. Während mein Interesse zunächst hauptsächlich literaturwissenschaftlichen Themen und Fragestellungen galt und ich meine Magisterarbeit zu den ideologiekritischen
Potenzialen in der Éducation sentimentale von Flaubert verfasst habe, ist meine Leidenschaft für die Kulturwissenschaft mit einem Seminar geweckt worden, das ich eher aus Verlegenheit besucht hatte. Es war den „Französischen Wegen zur Kulturwissenschaft" gewidmet und wurde von Dorothee Röseberg für Studierende der höheren Semester angeboten. Den Ausgangspunkt bildete schon damals die umstrittene Frage, ob es in Frankreich Kulturwissenschaft gibt. Im Zentrum standen dabei Einzelfallstudien von Vertretern der französischen Soziologie, der Geschichtswissenschaft und der Philosophie bzw. Literaturwissenschaft, die wir hinsichtlich ihrer kulturwissenschaftlichen Beiträge zur französischen und internationalen Diskussion untersucht haben. Im Nachhinein war es die Einübung eines, anderen‘ Blickes auf die Gegenstände, die mich nachhaltig fasziniert hat, zumal ich einigen der behandelten Denker bereits in anderen Kontexten begegnet war. Seither bin ich der Kulturwissenschaft, wie ich sie in Halle kenngelernt habe, verbunden geblieben.

\section{CM:}

Es waren sicherlich die Geschichtsseminare von Hélène Rioux in der Vorbereitungsklasse für die Aufnahmeprüfung der École normale supérieure, die mich an die histoire culturelle herangeführt haben. Einen besonderen Platz in den Lehrveranstaltungen, die für viele Schüler zum prägenden Erlebnis wurden, nahm dabei nicht zuletzt das Kino ein. Später habe ich mich an der Universität Paris 1 in den Maîtrise-Studiengang der Darstellenden Künste eingeschrieben, um unter der Leitung von Jean Gili über Filmgeschichte zu arbeiten. Verschiedene Reisen nach Berlin, die ich ab Mitte November 1989 unternommen hatte, haben mich dazu veranlasst, mein Forschungsterrain auf ostdeutsche Fiktionsfilme zu verlagern. Schließlich haben sich mein Blick und mein Forschungsansatz allmählich verändert. Ich habe mich, ausgehend von den Untersuchungen zur Filmgeschichte, mit dessen Eingliederung in die internationalen Beziehungen beschäftigt, um letztlich zur Erforschung der Geschichte des Internationalen Leipziger Festivals für Dokumentarfilm zu gelangen. Somit hat sich mein Forschungsansatz von einer histoire des représentations, einer auf die symbolischen Performanzen gestützten Geschichte, hin zu einer Geschichte verlagert, die das Kino aus einer institutionellen, wirtschaftlichen, sozialen und ästhetischen Perspektive untersucht. Die Ergebnisse liegen in meiner 2005 an der Universität Paris 1 verteidigten Doktorarbeit vor, die von Robert Frank und Etienne François betreut worden ist. Im Jahr 2006 hatte ich das große Glück, an die Universität Versailles Saint-Quentinen-Yvelines berufen zu werden und zum Mitglied des Centre d'histoire culturelle des sociétés contemporaines (CHCSC) zu avancieren, das 1992 von Jean-Yves Mollier und Pascal Ory gegründet worden war und zu jener Zeit unter der Leitung von Christian Delporte stand. Die Aufnahme in das CHCSC war ein entscheidender Schritt für die Ausrichtung meiner historiographischen Forschung, insbesondere im Hinblick auf die Mediengeschichte. So habe ich mich am Ende 
in einem Forschungszentrum wiedergefunden, in dem sich neben Historiker_innen auch Literaturwissenschaftler_innen, Forscher_innen in Information und Kommunikation und sogar Biolog_innen versammelt haben! Aus dieser Erfahrung heraus habe ich mir eine offene Konzeption der histoire culturelle angeeignet.

\section{WELCHE WERKE WÜRDEN SIE ALL JENEN EMPFEHLEN, DIE SICH MIT DER KULTUR- WISSENSCHAFT BZW. MIT DER HISTOIRE CULTURELLE I DEN ÉTUDES CULTURELLES BESCHÄFTIGEN MÖCHTEN?}

DR:

- Nünning, Ansgar / Nünning, Vera (2008), Einführung in die Kulturwissenschaften, Stuttgart, Metzler.

- Stierstorfer, Klaus / Volkmann, Laurenz (Hg.) (2005), Kulturwissenschaft interdisziplinär, Tübingen, Gunter Narr.

- Milling, Hanna (2010), Das Fremde im Spiegel des Selbst. Deutschland seit dem Mauerfall aus Sicht französischer, italienischer und spanischer Deutschlandexperten, Berlin, Logos.

- Cohen, Évelyne / Goetschel, Pascale / Martin, Laurent / Ory, Pascal (dir.) (2011), Dix ans d'histoire culturelle, Villeurbanne, Presses de l'Enssib.

- Poirrier, Philippe (2004), Les enjeux de l'histoire culturelle. Paris, Seuil.

- Ory, Pascal (2004), L'histoire culturelle, Paris, PUF.

FK:

Zusätzlich zu denen, die Dorothee Röseberg anführt, würde ich folgende nennen:

- Jaeger, Friedrich / Liebsch, Burkhard / Rüsen, Jörn / Straub, Jürgen (Hg.) (2011), Handbuch der Kulturwissenschaften, Bd. 1, Grundlagen und Schlüsselbegriffe, Stuttgart, Metzler.

- Jullien, François (2008), De l'universel, de l'uniforme, du commun et du dialogue entre les cultures, Paris, Fayard.

- Mäder, Marie-Therese / Metzger, Chantal / Neubert, Stefanie et alii (Hg.) (2016), Brücken bauen - Kulturwissenschaft aus interkultureller und multidisziplinärer Perspektive, Bielefeld, transcript.

- Morin, Edgar (1990), Introduction à la pensée complexe, Paris, Seuil.

MTM:

- Böhme, Hartmut / Matussek, Peter (2002), Orientierung Kulturwissenschaft. Was sie kann, was sie will, Reinbek, Rowohlt.

- Röseberg, Dorothee (2001), Kulturwissenschaft Frankreich, Stuttgart, Klett.
- Durand, Béatrice / Neubert, Stefanie / Röseberg, Dorothee / Viallon, Virginie (2006), Studieren in Frankreich und Deutschland. Akademische Lehr- und Lernkulturen im Vergleich, Berlin, Avinus.

- Müller-Funk, Wolfgang (2016), Theorien des Fremden. Eine Einführung, Tübingen, A. Francke Verlag.

- Goetschel, Pascale / Loyer, Emmanuelle 2011 [2002], Histoire culturelle de la France. De la belle Époque à nos jours, Paris, Armand Colin.

- Chalard-Fillaudeau, Anne (2015), Les études culturelles, Saint-Denis, Presses Universitaires de Vincennes.

CM:

Neben den bereits von meinen Kolleginnen genannten Arbeiten und dem unumgänglichen Dictionnaire d'histoire culturelle de la France contemporaine, herausgegeben von Christian Delporte, Jean-Yves Mollier und Jean-François Sirinelli (Paris, PUF, 2010) sowie den beiden Bänden zur französischen Kulturgeschichte vom 19. Jahrhunderts bis zur Gegenwart von Jean-Claude Yon, Pascale Goetschel und Emmanuelle Loyer, erschienen bei Armand Colin (2010 und 2002), möchte ich gerne, unter vielen anderen Werken, drei spezifischere Studien nennen, deren Autor_innen zwar nicht unbedingt der histoire culturelle angehören, die ich aber dennoch wärmstens empfehle:

- Gabilliet, Jean-Paul (2005), Des Comics et des hommes : histoire culturelle des comic books aux EtatsUnis, Nantes, Editions du Temps.

- Lindeperg, Sylvie (2007), Nuit et brouillard. Un film dans I'histoire, Paris, Odile Jacob.

- Mrozek, Bodo (2019), Jugend-Pop-Kultur. Eine transnationale Geschichte, Berlin, Suhrkamp.

\section{WARUM EINE DEUTSCH-FRANZÖSISCHE ZEITSCHRIFT INS LEBEN RUFEN?}

DR:

In Deutschland ist noch immer die Meinung verbreitet, dass es in Frankreich keine Kulturwissenschaft gäbe. In Frankreich kennt man nur in kleinen Kreisen die Entwicklungen der Kulturwissenschaften in Deutschland. Zugleich fehlt es bislang an einer Plattform für Debatten um theoretische und methodologische Fragen, die erhellen, ob und inwieweit das, was in Frankreich unter dem Siegel histoire culturelle und/oder études culturelles bezeichnet wird, äquivalent zu dem ist, was in Deutschland als Kulturwissenschaften bezeichnet wird. Außerdem geht es darum zu klären, wie sich die Entwicklungen in Frankreich und in Deutschland zu den international verbreiteten Cultural Studies verhalten. Eine deutsch-französische Verständigung über diese Frage ist längst überfällig und soll durch eine weitere internationale Öffnung der Zeitschrift ausgeweitet werden. 


\section{FK:}

In der Zeitschrift können Forschungspraktiken legitimiert, systematisiert und problematisiert werden, die bislang oftmals intuitiv und punktuell geblieben sind. Sie kann zeigen, wie Interdisziplinarität und Pluridisziplinarität in Kulturforschungen funktionieren und mit welchen Designs sie vorgehen. Ausgehend von einer pluralen Interpretation der Kulturwissenschaften wird es möglich sein, auch die Pluralität der präexistierenden Disziplinen und ihrer Grenzen zu formulieren wie auch ihre Übereinstimmungen zu ergründen. Dies erlaubt auch einen reflexiven und vertiefenden Umgang mit der eigenen Disziplin, was vor allem angesichts der Entwicklung der Disziplinen selbst besonders zweckdienlich ist. Als Vorbilder gelten für mich die Schriften und die Praxis des Philosophen/Soziologen Bruno Latour, der mit Spezialisten der Naturwissenschaften zusammenarbeitet, oder diejenigen von Yves Michaud, dem der Aufbau einer Universität „allen Wissens“ anvertraut wurde. Die Zeitschrift ist in ihren Rubriken offen sowohl für fiktionale als auch für politische Diskurse und informiert über Publikationen, die beispielhaft für wissenschaftliche Kooperationen sind, insbesondere - aber nicht nur - zwischen deutschen und französischen Forscher_innen, weil dies wegen unserer beruflichen Erfahrungen besonders einfach organisiert werden kann.

\section{MTM:}

Auch wenn das franco-allemand in aller Munde ist, sollte diese Tatsache nicht darüber hinwegtäuschen, dass es noch viel Potenzial auf dem Gebiet der gegenseitigen Verständigung zu Fragen nach den methodischen und konzeptuellen Ausrichtungen kulturwissenschaftlicher Forschung gibt. Zwar könnte man eine Reihe von Initiativen aufzählen, die sich um einen deutsch-französischen Wissenschaftsdialog bemühen, doch werden nur selten die epistemologischen Grundfragen der Kulturforschung zur Sprache gebracht. Die Zeitschrift bietet im besten Falle einen produktiven Diskussions- und Vorstellungsraum für Debatten und Projekte aus Wissenschaft und Praxis, der disziplinäre und nationale Grenzen überwindet.

\section{CM:}

Die deutsch-französischen Beziehungen sind sowohl zu einer Selbstverständlichkeit als auch zu einer neuen Herausforderung geworden, der es sich zu stellen gilt. Tatsächlich gehören die deutsch-französischen Projekte in der Forschung, in den Geistes- und Sozialwissenschaften wie auch in anderen Disziplinen, zum integralen Bestandteil auf dem Gebiet der internationalen Kooperationen (Dank der Unterstützung solcher Schlüsselinstitutionen wie dem DAAD, dem CIERA oder dem Centre Marc Bloch in Berlin). Zugleich ist offenkundig, dass das Wissen über die wissenschaftliche Produktion des Nachbarlandes nicht immer sehr groß ist und die Studierenden wie auch die Forscher_innen, die keine Frankreich- bzw. Deutschlandspezialist_innen sind, noch eher die Arbeiten der anglophonen Kolleg_innen kennen. Angesichts dieser mangelnden gegenseitigen Kenntnisnahme, die nicht nur die Spezialist_innen im engeren Sinne betrifft, die sich mit den besagten Nachbarländern beschäftigen, scheint es notwendig, einen Ort vorzuschlagen, an dem einschlägige aber noch nicht übersetzte Arbeiten präsentiert werden können. Damit versteht sich diese Zeitschrift als Instrument, um im Rahmen des franco-allemand $z u$ arbeiten, ohne jedoch eine allzu enge Auffassung von dieser Herangehensweise zu vertreten. Die europäische und internationale Dimension soll dabei nicht ausgeschlossen werden. Auch sollten die Forschungen auf den Gebieten der histoire culturelle / études culturelles / Kulturwissenschaften in aller Deutlichkeit auf die Gesamtheit der französisch- und deutschsprachigen Räume übertragen werden. In diesem Sinne gehört die Zirkulation der Ideen, und mit ihr jene der Studierenden und Forscher_innen, zu den Hauptanliegen unserer Zeitschrift.

\section{WELCHE ERFAHRUNGEN HABEN SIE SELBST MIT DEM DEUTSCH-FRANZÖ- SISCHEN?}

DR:

Als deutsche Frankreichforscherin und Universitätsprofessorin habe ich nicht nur über 30 Jahre wissenschaftlich zu den deutsch-französischen Kulturkontakten, zum Kulturvergleich und -transfer, meist in Kooperation mit französischen Kolleg_ innen, gearbeitet und publiziert, sondern auch in zahlreichen deutsch-französischen Gremien, darunter im wissenschaftlichen Beirat der DFH und im DAAD gearbeitet, mit AnneMarie Pailhès einen deutsch-französischen Studiengang an der MLU Halle und an der Universität Paris Nanterre eingerichtet und geleitet sowie Promovend_innen im Rahmen einer cotutelle zur Promotion in Deutschland und in Frankreich geführt.

\section{FK:}

Meine Erfahrungen mit dem Deutsch-Französischen begannen mit einem Studium, das gleichermaßen Sprachgeschichte und Kunst verband und dabei den Konflikten, in denen Frankreich Deutschland und Österreich gegenüberstand, besondere Aufmerksamkeit widmete. Als ich etwas später Gelegenheit hatte, präzisere Untersuchungen zu den Kriegen und den Friedensverträgen vorzunehmen, um dabei genauer und direkter die Kontinuitäten und die Zäsuren zu analysieren, die diese herbeigeführt hatten, war es möglich geworden, verbreitete Vereinfachungen, die mit so zentralen Begriffen wie deutsch-französische Freundschaft und deutsch-französische Aussöhnung verbunden sind, aufzuspüren und offenzulegen. 
MTM:

Das franco-allemand begleitet mich seit meiner Schulzeit am Französischen Gymnasium in Berlin und ist seither zu einem festen Bestandteil in meinem privaten und beruflichen Alltag geworden. In besonderer Erinnerung bleiben mir die deutsch-französischen Nachwuchsseminare des CIERA in Frankreich und die gemeinsame Zusammenarbeit mit Dorothee Röseberg und Anne-Marie Pailhès im Rahmen des bereits erwähnten Doppelstudiengangs an den Universitäten Halle und Nanterre. Ein weiterer Höhepunkt war die gemeinsam mit Jenny Ettrich organisierte Sektion auf dem Frankoromanistentag in Saarbrücken. Im Ergebnis sind deutsche und französische Romanist_innen, German_istinnen, Historiker_innen sowie Soziolog_innen zusammengekommen, um über die Potenziale kulturwissenschaftlicher Forschung in den jeweiligen Disziplinen und Ländern zu diskutieren.

\section{CM:}

Ich bin erstmals während meines Aufenthaltes am Centre Marc Bloch im Jahr 1994 in die Welt des franco-allemand eingetaucht; zu jenem Zeitpunkt also, als ich meine Untersuchungen für die Maîtrise durchgeführt habe. Seitdem arbeite ich regelmäßig mit Forscher_innen deutscher Institutionen zusammen, wie etwa dem ZZF in Potsdam, wo ich das Glück hatte, zweimal als Gastwissenschaftlerin eingeladen zu werden. Aus diesen Zusammenarbeiten haben sich Studientage, Kongresse sowie Publikation in deutscher oder englischer Sprache ergeben. Darüber hinaus habe ich zwei Jahre lang am Frankreichzentrum der Universität Leipzig unterrichtet. Meine zahlreichen Forschungsaufenthalte in Deutschland wurden größtenteils durch Institutionen finanziert, die im Zentrum der deutsch-französischen Forschung stehen: das CIERA, der DAAD und das Centre Marc Bloch. Für mich ist die Realität des franco-allemand jedoch nicht alleine an die Institutionen oder den Beruf gebunden, sie ist vielmehr an den Alltag und die Familie gekoppelt, da mein Mann Deutscher ist und wir sowohl in Paris gelebt haben als auch in Köln oder Berlin.

\section{WELCHE WÜNSCHE HABEN SIE FÜR DIE ZEITSCHRIFT?}

\section{DR:}

Mit der Gründung der Zeitschrift verbindet sich für mich ein seit langem gehegter Wunsch: mit wissenschaftlich Gleichgesinnten in Frankreich, in Deutschland und darüber hinaus zu arbeiten, ohne Rechtfertigungsnot und auf einer eigens dafür gegründeten kulturwissenschaftlichen Plattform Hypothesen und Arbeitsergebnisse auszutauschen. Die Zeitschrift soll in ihrer Offenheit für verschiedene Konzepte dazu beitragen, die Produktivität kulturwissenschaftlichen
Arbeitens über nationale Grenzen hinaus zu verdeutlichen und es möge gelingen, durch die konzeptionelle Ausrichtung den Vorwurf der Beliebigkeit zu entkräften. Die Zeitschrift soll die Vorzüge eines weiten und nicht normativen Kulturbegriffs überzeugend vertreten. Und sie soll vor allem auch eines sein: eine Provokation für etablierte Disziplinen, verbunden mit der Aufforderung, das Überschreiten von Grenzen zuzulassen.

\section{FK:}

Da wir im Vorfeld lange über die Orientierungen und Ziele dieser Zeitschrift diskutiert haben, möchte ich mich ganz den von Dorothee Röseberg gegebenen Antworten anschließen. Persönlich liegt mir viel daran zu unterstreichen, dass ich es im Vorfeld oft als Schwierigkeit empfand, die Pluridisziplinarität in der Sprach- Literatur- und Kunstwissenschaft zu theoretisieren, obgleich ich eine solche Theoretisierung für notwendig erachte. Ohne theoretische Grundlagen besteht das Risiko der pluridisziplinären Arbeit in einer Anhäufung von Forschungen, einem Nebeneinanderstellen von Themen und schließlich einer Heterogenität, die der Effektivität und Nützlichkeit der Kooperationen schadet. Die Zeitschrift wird Lösungen vorschlagen, die einer solch bloßen Anhäufung als Hürde für effektive Kooperationen vorbeugen können. In diesem Sinne werden konzeptionelle Instrumente vorgestellt und durch die Präsentation von Varianten bereichert, die sich in verschiedenen kulturellen Räumen entwickelt haben. Zugleich werden Untersuchungsobjekte polysemischen Forschungen unterzogen, die zugleich unter ästhetischen Gesichtspunkten - die mir sehr wichtig sind wie auch historischen, sozialen und politischen Aspekten untersucht.

\section{MTM:}

Ich wünsche mir, dass die Zeitschrift zu einem lebendigen Ort des Austausches und der Inspiration für jüngere Wissenschaftler_innen wird. Sie sollen dazu ermuntert werden, sich auch mit ungewöhnlichen Themen und Konzepten zu beschäftigen, die vielleicht aus dem Rahmen der disziplinären Grenzen und gewohnten Denkschemata fallen, aber dafür neue Formen kulturwissenschaftlicher Forschung ausloten.

\section{CM:}

Ich wünsche mir, dass die Zeitschrift zu einem Ort des Austausches und des Ausdrucks für jüngere Forscher_innen wird, die das gemeinsame Interesse vereint, über ihren gewohnten wissenschaftlichen Horizont zu blicken; ob sie nun aus Frankreich und Deutschland oder aber aus anderen Ländern kommen. Einzig solch eine Öffnung ermöglicht es, einschlägige Forschungsthemen zu generieren bzw. zu entwickeln und auf diese Weise einen Beitrag zu einem qualitätsvollen deutsch-französischen und europäischen Raum zu leisten. 


\section{Entretien avec les éditrices}

\section{QUELS SONT VOS OBJETS DE RECHERCHE ACTUELS ?}

\section{DR:}

L'ouvrage que je viens de publier aux éditions Narr (2018) est une biographie que j'ai consacrée, en choisissant une approche culturelle, à Rita Schober, spécialiste de Zola et dont la réputation a été internationale. II montre dans quelle mesure les ressources des recherches en sciences des cultures sont transposables à l'édition historique et critique d'une (auto)biographie. Das Fremde als Anspruch sera le titre d'un article théorique sur les sciences des cultures qui va paraître très prochainement dans un ouvrage collectif, Interkulturalität und Migration, et qui examine dans quelle mesure notions phénoménologiques et théories des systèmes sont applicables à l'interprétation scientifique des expériences de l'altérité. Je travaille en outre depuis plusieurs années au vaste champ de recherche que sont Bildung vs formation de la raison? L'objectif est de traiter des convergences et des différences entre les concepts et les pratiques d'enseignement, d'apprentissage et d'étude dans les institutions éducatives allemandes et françaises. Un des aspects primordiaux de cette enquête comparative sera de voir comment des notions abstraites provenant de l'histoire des idées peuvent s'associer à des pratiques, puis déboucher, en fonction des configurations sociales, sur l'acquisition d'habitudes et ainsi finir par contribuer à former les mentalités de certains groupes. Cela implique que l'histoire des idées soit reliée à la sociologie et que la perspective comparatiste soit complétée par l'approche interculturelle. Cette dernière gagnera en valeur heuristique dans la mesure où seront ici commentés les constats empiriques de malentendus ou d'obstacles rencontrés lors d'études faites à l'étranger. Studieren in Frankreich und Deutschland, une approche comparée des cultures universitaires (2006 allemand, 2007 français) et Formation de la raison (2012) servent de socle à d'autres études de cas qui appliquent cette grille culturelle; à ce stade, mes recherches se concentrent pour l'instant sur l'époque de la Monarchie de juillet en France.

\section{FK:}

Des diables et des spectres: croyances et jeux littéraires, l'avant-dernière publication collective que j'ai codirigée, a l'objectif de signaler la porosité entre position des élites et pratiques populaires face à la question de la superstition combattue par les élites intellectuelles et en définitive diversement instrumentalisée; cette publication examine le basculement épistémologique qui s'est produit autour de 1800, a eu des répercussions idéologiques, culturelles, esthétiques, a rendu possible le jeu littéraire moderne s'amusant des diables et des spectres et a aussi induit l'interrogation sur la responsabilité voire la culpabilité de l'homme moderne face au mal appréhendé désormais de façon sécularisée.

La publication collective suivante porte sur "L'art épistolaire entre civilité et civisme» de Gellert à Grass : elle examine l'évolution de la gradation qui s'est établie, dans, par ou pour l'épistolaire, entre civilité et civisme entre le XVIIle et le XXe siècle, à partir du moment où le discours épistolaire allemand tend à s'émanciper de modèles français. L'épistolier a été appréhendé ici en fonction de son insertion dans la communauté, que celle-ci ait été un environnement social, préexistant et imposé, ou affectif et librement choisi. La civilité épistolaire a acquis une dimension sociale et philosophique, puis a tenu compte du changement de paradigmes anthropologiques au début du XIXe siècle, simulant alors la diversification des types de lettres, la démultiplication des usages rhétoriques et un militantisme politique ou social.

Une de mes recherches actuelles se situe dans le prolongement de ces réflexions: la correspondance de l'archiduc Jean d'Autriche, qui est restée en grande partie inédite et dont les réflexions sur «le peuple de Styrie» nous apprennent beaucoup sur la représentation que cet archiduc, pionnier de la Volkskunde, se faisait de l'éducation citoyenne. Un autre projet est l'édition commentée des relations de voyages de Johann Georg Sulzer, un volume étant prévu sur ces textes dans le cadre de la publication de ses œuvres complètes (Schwabe Verlag). Enfin, j'ai l'intention de continuer à travailler sur des auteurs-voyageurs, sur leur confrontation à des communautés étrangères et leur expérience de l'altérité, eux qui sont porteurs de normes issues de leur culture d'origine, notamment philosophiques et religieuses, et de ce fait particulièrement sensibles aux processus d'intégration ou d'exclusion dans les sociétés visitées.

\section{MTM :}

Ma thèse, que j'ai soutenue en 2017, était consacrée à la culture du savoir des élites françaises à la fin du XVIIle siècle (Königshausen \& Neumann 2019). Ce travail s'inscrivait dans un projet plus large portant sur ce que Dorothee Röseberg a nommé le modèle culturel de la formation de la raison. II s'agit d'un modèle construit autour du concept central du raisonnement, qui donne priorité à certaines opérations de la pensée. Contrairement à ce que la recherche jusqu'à présent a démontré, ce modèle s'avère s'être constitué à partir du rationalisme et du sensualisme des Lumières et avoir pu s'institutionnaliser après la Révolution dans le cadre des pratiques éducatives conçues pour les élites françaises et assorties à ce titre d'une ambition nationale et modélisante. Mes recherches s'intéressent 
aux réseaux institutionnels des Idéologues et j'ai pu montrer combien ces derniers ont joué un rôle essentiel pour la pérennisation de ce modèle culturel. J'ai présenté les premiers résultats de mon étude dans les actes du colloque que j'ai publiés avec Jenny Ettrich et intitulés Dialogpotenziale kulturwissenschaftlicher Forschung in den Fremdsprachenphilologien (Peter Lang 2018). Je suis actuellement postdoctorante à l'université de Brême où je coordonne le projet de recherche soutenu par la DFG (Deutsche Forschungsgemeinschaft) „La circulation et la transformation transatlantiques des idées: la réception des Lumières dans la littérature franco-caraïbéenne contemporaine". Je m'intéresse, dans une perspective d'études culturelles et transnationales, aux processus de circulation des savoirs et des discussions au sujet de la nature dans les textes francophones caribéens du XVIIle à nos jours.

\section{$\mathrm{CM}$ :}

Un premier ensemble de recherches menées depuis plusieurs années porte sur les festivals internationaux de cinéma dans l'Europe de la guerre froide. Le point de départ de ces recherches a été l'étude du festival international de films documentaires de Leipzig en RDA, créé en 1955 et qui continue d'avoir lieu chaque année. A travers ces manifestations festivalières, j'étudie les logiques de circulations internationales et transnationales qui ont existé, malgré les divisions diplomatiques, entre l'Est et l'Ouest mais aussi le Nord et le Sud, en analysant tout à la fois les institutions, plusieurs trajectoires individuelles et les films eux-mêmes, au cœur de ces échanges (Cinéma et guerre froide, Paris, 2014 et la traduction en anglais, Screened Encounters, New York, 2018) Dans la continuité de ces enjeux, nous avons lancé, avec quelques collègues, français, allemands et italiens, un projet d'histoire croisée des écoles de cinéma européennes et non-européenne, de l'entre-deux-guerres jusqu'aux années 1990.

Un deuxième ensemble de travaux, plus récents et au coeur de mes recherches actuelles, porte sur l'histoire en Europe des mouvements de solidarité internationale avec le Chili après le coup d'Etat militaire du 11 septembre 1973 et l'arrivée au pouvoir de la junte militaire. Un de mes axes de recherche est consacré au parcours et à l'engagement du pasteur ouest-allemand Helmut Frenz (1933-2011), figure clé de la solidarité entre Amérique latine et Europe.

\section{COMMENT ÊTES-VOUS VENUE À L'HISTOIRE CULTURELLE / AUX ÉTUDES CULTURELLES / AUX KULTURWISSENSCHAFTEN?}

\section{DR:}

II me serait possible de répondre en relatant les nombreux obstacles rencontrés car, dans la romanistique traditionnellement conçue comme Geisteswissenschaft (discipline littéraire et philologie des langues étrangères), il n'y avait pas de Kulturwissenschaft, et encore de nos jours cette dernière n'est pas partout entrée dans les mœurs. Un tel constat vaut en particulier si l'on voit dans la Kulturwissenschaft une passerelle entre Geisteswissenschaft et sciences sociales, comme c'est le cas dans mes propres travaux. Tout a commencé à l'université Humboldt où, durant mes études, j'avais pu bénéficier de coopérations avec des historiens et eu accès aux recherches menées par l'Institut für Kulturwissenschaften. Malgré les restrictions idéologiques et matérielles que la RDA imposait aux chercheurs, je peux dire rétrospectivement que j'ai continuellement travaillé «en nageant entre deux eaux ", à la marge des disciplines institutionnellement établies, voire en transgressant leurs frontières. La conséquence en a été que mes recherches ont longtemps été publiées dans des revues et ouvrages relevant de disciplines fort diverses. Mon mémoire de maîtrise, encadré par des historiens, analysait le discours des médias, et ce à propos du retour au pouvoir de Charles de Gaulle en 1958. Recrutée comme assistante, j'ai participé à des projets de recherches littéraires mais au demeurant assez exotiques: études sur l'histoire des fonctions de la littérature pour les élites françaises, sur les canons littéraires en France, sur la sociologie de la littérature à partir d'analyses empiriques comparées. Ma nomination sur une chaire professorale dédiée aux sciences des cultures dans les pays de langues romanes a correspondu à une deuxième période de ma carrière, avec, sur le plan des objets de recherche, une émancipation du champ purement littéraire et, sur le plan de la théorie et de la méthodologie, une spécialisation en sciences des cultures appliquées aux philologies étrangères. II est important, selon moi, d'expliciter quel est le regard qui est porté sur l'objet qu'on analyse. Les concepts permettant de cerner différentes formes des contacts entre cultures et leurs résultats, que ce soit dans les médias ou en situation de rencontres, ainsi que le problème de l'altérité ou de l'expérience de l'étranger sont au centre de mes recherches. Ma définition de la culture n'est pas normative, elle s'appuie sur les pratiques culturelles et a ainsi pris une orientation socio-anthropologique. Je n'en suis pas moins restée une philologue qui n'entend pas négliger les composantes linguistiques de la communication.

\section{FK:}

Dans le cas d'une germaniste française, ce chemin est en fait cohérent. C'est d'abord dans le cadre de mes recherches sur les relations de voyage allemandes et françaises du XVIIle siècle qu'il m'a fallu chercher une légitimation scientifique pour appréhender un type de discours qui, à l'époque, n'avait pas encore acquis ses lettres de noblesse en raison de son hybridité. A cela s'ajoutait une autre spécificité, celle de l'évolution du discours et son ajustement à chaque génération ; en effet, si la tradition des voyageurs consista, même après 1800 , à transmettre des données géographiques, économiques, sociologiques, les grilles de perception évoluaient périodiquement, celles du XIX ${ }^{e}$ siècle impliquant de prendre de plus en plus en considération la vie culturelle populaire et affinant 
l'appréhension des paysages et l'expression de la sensibilité religieuse, et faisant finalement triompher l'émancipation du sujet. La notion d'histoire des mentalités était, à l'époque où je menais ces recherches, celle qui paraissait le plus opératoire. II est pour moi évident que celle d'histoire culturelle est plus adéquate au genre de la relation de voyage car elle offre les catégories utiles pour évaluer les qualités discursives, les assertions des auteurs sur leur identité, le degré de leur représentativité et l'impact de leur bagage culturel.

C'est ensuite dans le cadre de mes activités d'enseignante, bénéficiant des avancées de la " civilisation » depuis le début des années 1990, que j'ai souhaité proposer aux étudiants et aux doctorants de travailler sur des supports textuels se situant au carrefour de la littérature et de l'histoire. Pour citer un exemple récent: les feuilletons journalistiques des années 1914-18 permettaient d'aborder la délicate question de la " culture de guerre » et d'étudier la créativité et la modernité de certains auteurs sans qu'il y ait divorce entre littérature et histoire.

\section{MTM :}

La voie qui m'a conduite à la Kulturwissenschaft est étroitement liée à mes études de franco-romanistique à l'université Martin Luther de Halle-Wittenberg. Alors que mon intérêt initial avait surtout porté sur les théories de la littérature et les questionnements afférents, de sorte que mon mémoire de Master a été consacré à l'Education sentimentale de Flaubert et au potentiel de ses critiques idéologiques, c'est ensuite un très vif intérêt que j'ai éprouvé pour la Kulturwissenschaft quand je l'ai découverte à l'occasion d'un séminaire auquel je m'étais inscrite plutôt par hasard. Ce séminaire s'intitulait «Approches françaises de la Kulturwissenschaft ॥; il était proposé par la professeure Dorothee Röseberg et ouvert aux étudiants en fin de cursus universitaire. Le point de départ était déjà la question de savoir si l'on pouvait parler en France d'une Kulturwissenschaft. Les textes que nous avions à examiner étaient les débats que des sociologues, des historiens, des philosophes français ou même des théoriciens de la littérature avaient engagés, à l'échelle française et internationale, à propos des sciences des cultures. Vu rétrospectivement, c'est l'étude du cadre herméneutique et la mise en perspective historique des sciences de la culture qui m'a durablement fascinée, d'autant que j'avais déjà travaillé sur certains de ces théoriciens. Depuis cette époque, la Kulturwissenschaft est restée au centre de mes recherches.

\section{CM:}

Ce sont sans doute les cours d'histoire d'Hélène Rioux en classe préparatoire qui m'ont initiée à l'histoire culturelle. Le cinéma tout particulièrement tenait une place importante dans ses enseignements, qui ont marqué nombre de ses élèves. Plus tard, je me suis inscrite en maîtrise en Etudes du spectacle à l'université Paris1 pour travailler sur l'histoire du cinéma, sous la direction de Jean Gili. Différents voyages à Berlin à partir de novembre 1989 m'ont conduit à choisir comme terrain de recherche les films de fiction est-allemands. Puis mon regard et ma démarche ont évolué. Des études d'histoire du cinéma, je suis passée en histoire des relations internationale, afin d'étudier l'histoire du festival de Leipzig. D'une histoire des représentations, je suis passée à une histoire où le cinéma était étudié sous l'angle institutionnel, économique, sociale et esthétique. La thèse sous la direction de Robert Frank et d'Etienne François a été soutenue en 2005 à Paris 1. En 2006 j'ai eu la grande chance d'être élue à l'Université de Versailles Saint-Quentin-en-Yvelines et de devenir membre titulaire du Centre d'histoire culturelle des sociétés contemporaines. Lancé en 1992, sous la houlette de Jean-Yves Mollier et Pascal Ory, le CHCSC était alors dirigé par Christian Delporte. L'intégration au CHCSC a été décisive pour situer davantage mon travail de recherche dans ce champ historiographique, notamment au sein de l'histoire des médias. Je me suis ainsi retrouvée dans un laboratoire avec des historiens, des littéraires, des infocommistes et même des spécialistes de biologie ! J'en ai acquis une conception ouverte de l'histoire culturelle.

\section{QUELS OUVRAGES CONSEILLERIEZ-VOUS DE LIRE À QUI SOUHAITE DÉCOUVRIR L'HISTOIRE CULTURELLE / ÉTUDES CULTURELLES / KULTURWISSENSCHAFTEN}

DR:

- Nünning, Ansgar / Nünning, Vera (2008), Einführung in die Kulturwissenschaften, Stuttgart, Metzler.

- Stierstorfer, Klaus / Volkmann, Laurenz (Hg.) (2005), Kulturwissenschaft interdisziplinär, Tübingen, Gunter Narr.

- Milling, Hanna (2010), Das Fremde im Spiegel des Selbst. Deutschland seit dem Mauerfall aus Sicht französischer, italienischer und spanischer Deutschlandexperten, Berlin, Logos.

- Cohen, Évelyne / Goetschel, Pascale / Martin, Laurent / Ory, Pascal (dir.) (2011), Dix ans d'histoire culturelle, Villeurbanne, Presses de l'Enssib.

- Poirrier, Philippe (2004), Les enjeux de l'histoire culturelle. Paris, Seuil.

- Ory, Pascal (2004), L'histoire culturelle, Paris, PUF.

FK:

Outre ceux signalés par D. Röseberg, voici quelques ajouts:

- Jaeger, Friedrich / Liebsch, Burkhard / Rüsen, Jörn / Straub, Jürgen (Hg.) (2011), Handbuch der Kulturwissenschaften, Bd. 1, Grundlagen und Schlüsselbegriffe, Stuttgart, Metzler.

- Jullien, François (2008), De l'universel, de l'uniforme, $d u$ commun et du dialogue entre les cultures, Paris, Fayard. 
- Mäder, Marie-Therese / Metzger, Chantal / Neubert, Stefanie et alii (Hg.) (2016), Brücken bauen - Kulturwissenschaft aus interkultureller und multidisziplinärer Perspektive, Bielefeld, transcript.

- Morin, Edgar (1990), Introduction à la pensée complexe, Paris, Seuil.

MTM :

- Böhme, Hartmut / Matussek, Peter (2002), Orientierung Kulturwissenschaft. Was sie kann, was sie will, Reinbek, Rowohlt.

- Röseberg, Dorothee (2001), Kulturwissenschaft Frankreich, Stuttgart, Klett.

- Durand, Béatrice / Neubert, Stefanie / Röseberg, Dorothee / Viallon, Virginie (2006), Studieren in Frankreich und Deutschland. Akademische Lehr- und Lernkulturen im Vergleich, Berlin, Avinus.

- Müller-Funk, Wolfgang (2016), Theorien des Fremden. Eine Einführung, Tübingen, A. Francke Verlag.

- Goetschel, Pascale / Loyer, Emmanuelle 2011 [2002], Histoire culturelle de la France. De la belle Époque à nos jours, Paris, Armand Colin.

- Chalard-Fillaudeau, Anne (2015), Les études culturelles, Saint-Denis, Presses Universitaires de Vincennes.

\section{CM :}

En plus des ouvrages indiqués par mes collègues, et des incontournables comme le Dictionnaire d'histoire culturelle de la France contemporaine, dirigé par Christian Delporte, Jean-Yves Mollier et Jean-François Sirinelli (Paris, PUF, 2010) ou les deux volumes d'histoire culturelle de la France du XIX à nos jours par Jean-Claude Yon, Pascale Goetschel et Emmanuelle Loyer chez Armand Colin (2010 et 2002), j'aimerais citer, parmi bien d'autres, trois études plus spécifiques, dont les auteurs ne se réclament pas obligatoirement d'histoire culturelle, mais dont je conseille chaleureusement la lecture:

- Gabilliet, Jean-Paul (2005), Des Comics et des hommes: histoire culturelle des comic books aux EtatsUnis, Nantes, Editions du Temps.

- Lindeperg, Sylvie (2007), Nuit et brouillard. Un film dans I'histoire, Paris, Odile Jacob.

- Mrozek, Bodo (2019), Jugend-Pop-Kultur. Eine transnationale Geschichte, Berlin, Suhrkamp.

\section{POURQUOI LANCER UNE REVUE FRANCO- ALLEMANDE?}

\section{DR:}

II est fréquent d'entendre dire, en Allemagne, que l'on n'étudierait pas les sciences des cultures en France.
Inversement, il n'y a en France que des petits cercles qui sont au courant des évolutions des sciences des cultures en Allemagne. En outre, faisant partie des desiderata de la recherche, une plate-forme ouverte aux débats théoriques et méthodologiques permettra d'examiner si et dans quelle mesure ce qu'on place en France sous le sceau de l'histoire culturelle et/ou des études culturelles est l'équivalent de ce qu'on entend en Allemagne par sciences des cultures. Les échanges à ce sujet sont nécessaires depuis bien longtemps, pour le franco-allemand comme pour d'autres pays, car la revue souhaite enrichir les réflexions grâce à une large ouverture à l'international.

\section{FK:}

La revue pourra légitimer, systématiser, problématiser des pratiques restées parfois intuitives et ponctuelles. Elle pourra analyser et désigner le fonctionnement de l'interdisciplinarité et de la pluridisciplinarité dans l'étude des cultures. Grâce à son interprétation des sciences des cultures, la pluralité de disciplines préexistantes sera respectée, les délimitations disciplinaires explicitées et les convergences exploitées. Cela permet aussi un retour et un approfondissement réflexif sur sa propre discipline, ce qui est d'autant plus pertinent que les disciplines sont elles-mêmes souvent en constante évolution. La revue ouvrira ses colonnes à des analyses de discours aussi bien fictionnalisés que politisés, et pourra informer sur les publications qui en fournissent des exemples, comme celles du philosophe sociologue Bruno Latour qui impulse des coopérations avec des spécialistes des sciences dures ou celles d'Yves Michaud, auquel fut confié le lancement de l'université de tous les savoirs.

Le fait d'associer les aires francophones et germanophones devrait aider à greffer les notions de représentation, interprétation, symbolique, sur des faits datés et localisés, à éviter les stéréotypes tout en mettant en relief des particularités culturelles, à se concentrer sur des thématiques communes sans céder à l'hétérogénéité, à proposer des grilles de lecture sans pour autant basculer dans le prescriptif, ouvrir un espace à l'élaboration de réflexions et de discussions.

\section{MTM :}

Bien que le franco-allemand soit à l'ordre du jour, il ne faut pas sous-estimer le fait qu'il reste beaucoup à explorer de part et d'autre afin de cerner les différences dans les approches que nous avons en matière de méthodologie et de conceptualisation. II serait certes possible de signaler toute une série de réalisations qui se placent au service d'un dialogue franco-allemand mais les fondements épistémologiques de la recherche sur les cultures y sont rarement explicités. La présente revue offrira dans tous les cas une plate-forme qui permettra des échanges et des présentations de discussions et de projets concernant le volet scientifique et pratique de ces recherches et qui dépassera les frontières nationales et disciplinaires. 
CM:

Les relations franco-allemandes sont devenues tout à la fois une évidence et un nouveau défi à relever. En effet, les projets franco-allemands dans le domaine de la recherche, des sciences humaines comme dans d'autres disciplines, sont partie prenante du champ des coopérations internationales (grâce au soutien notamment d'institutions clés comme le DAAD, le CIERA ou le Centre Marc Bloch à Berlin). Dans le même temps, il faut bien reconnaître que la connaissance de la production scientifique du pays voisin n'est pas toujours très grande et les étudiants comme les chercheurs non spécialistes de la France / de l'Allemagne connaissent davantage les travaux des collègues anglophones. Face à cette méconnaissance par un cercle plus large que celui des spécialistes du champ de ce qui se fait outre-Rhin, il semble nécessaire de proposer un lieu où soient présentés des travaux pertinents mais non encore traduits. La revue se veut donc être le moyen de travailler dans un cadre franco-allemand mais audelà d'une vision trop étroite de cette approche. La dimension européenne et internationale ne saurait être exclue, ne seraitce qu'en intégrant de toute évidence les recherches en histoire culturelle / études culturelles / Kulturwissenschaften dans l'ensemble des espaces francophones et germanophones. Encourager la circulation des idées, et donc des étudiants et des chercheurs, est le principal objectif de notre revue.

\section{QUELLE EST VOTRE PROPRE EXPÉRIENCE DU FRANCO-ALLEMAND?}

\section{DR:}

En tant qu'Allemande franco-romaniste et professeure d'université, cela fait plus de trente ans que je travaille et publie des études scientifiques sur les contacts culturels francoallemands et sur les comparaisons et transferts culturels, le plus souvent en coopération avec des collègues français ainsi que dans de nombreux comités franco-allemands, notamment dans le Conseil scientifique de l'UFA et au DAAD. J'ai fondé et co-dirigé avec Anne-Marie Pailhès un parcours universitaire bi-diplômant entre les universités de Halle et de Paris Nanterre.

\section{FK:}

Mon expérience du franco-allemand avait commencé par l'étude conjointe et paradoxale de l'histoire de la langue, de l'art ainsi que des conflits ayant opposé la France à l'Allemagne et à l'Autriche. Avoir eu par la suite l'occasion de mener des recherches plus précises et d'alors traiter les guerres et les traités de paix pour eux-mêmes, d'analyser de plus près les continuités et les césures qu'ils entraînent, a permis de dépister les simplifications de notions telles que celle d'amitié et de réconciliation.
MTM :

Ma scolarité s'étant effectuée au Lycée français de Berlin, je suis depuis longtemps familiarisée avec le franco-allemand, et ce à titre personnel tout comme sur le plan de ma carrière professionnelle. J'ai en particulier beaucoup apprécié les séminaires organisés par le CIERA pour former la jeune génération à la recherche ainsi que la coopération avec les professeurs Dorothee Röseberg et Anne-Marie Pailhès dans le cadre du programme franco-allemand (parcours universitaire bi-diplômant) des universités de Halle et de Nanterre. En 2016, lors du Congrès des franco-romanistes qui a eu lieu à Sarrebruck, j'ai eu en outre le grand privilège de piloter, avec Jenny Ettrich, une section dédiée spécifiquement aux sciences des cultures. Les philologues spécialistes de langues étrangères, en l'occurrence surtout des romanistes allemands et des germanistes français, ainsi que les historiens et les sociologues des deux pays qui y ont participé ont discuté des ressources que la recherche en matière de sciences des cultures pouvait apporter à leurs disciplines et dans leurs pays respectifs

\section{CM :}

C'est lors de mon premier séjour au Centre Marc Bloch, en 1994, pour mes recherches en maîtrise, que j'ai plongé dans le monde du franco-allemand. Depuis lors, je travaille régulièrement avec des chercheurs d'institutions allemandes, dont le ZZF à Potsdam, où j'ai eu la chance d'être deux fois accueillie comme chercheure invitée: journées d'étude, colloques, publications en allemand ou en anglais ont résulté de ces collaborations. J'ai par ailleurs enseigné deux années au Frankreich Zentrum de l'Université de Leipzig. Mes nombreux séjours de recherche en Allemagne ont été en grande partie financés par des institutions au cœur de la recherche franco-allemande: le CIERA, le DAAD et le Centre Marc Bloch. Mais, pour moi, la réalité du franco-allemand n'est pas qu'une histoire institutionnelle et professionnelle, c'est aussi une réalité quotidienne et familiale puisque mon mari est allemand et que nous avons habité aussi bien à Paris qu'à Cologne ou Berlin.

\section{QUELS SONT VOS SOUHAITS POUR LA REVUE?}

\section{DR:}

Fonder une telle revue est un vœu que j'ai formulé depuis longtemps: l'objectif est de travailler avec des collègues partageant mes convictions, que ce soit en France, en Allemagne ou au-delà, sans qu'il soit nécessaire de se légitimer, afin d'échanger nos hypothèses et conclusions sur une plate-forme dédiée spécialement aux sciences des cultures. La présente revue devra, en respectant la pluralité 
des concepts, contribuer à mettre en évidence la productivité des recherches en sciences des cultures et, en insistant sur les enjeux conceptuels, s'efforcer d'éviter l'écueil du fourretout thématique. Elle devra illustrer les avantages qu'offre la notion de culture si elle est prise dans un sens large et non normatif. Et elle devra avant tout ne pas reculer devant l'objectif de provoquer les disciplines qui resteraient repliées sur elles-mêmes et inciter à décloisonner les frontières disciplinaires.

\section{FK :}

Comme nous avons préalablement longuement réfléchi avec Dorothee Röseberg sur les orientations et les objectifs de cette revue, je me rallie totalement à la réponse qu'elle vient de donner. J'ajoute que, à titre personnel, j'avais précédemment trouvé difficile de théoriser la pluridisciplinarité en arts, lettres et langues, alors que cette théorisation est absolument nécessaire. Sans des repères théoriques, le risque récurrent des travaux pluridisciplinaires est un empilement d'investigations scientifiques, une juxtaposition de thèmes, et pour finir une hétérogénéité qui nuit à l'efficacité voire même à l'utilité des coopérations. La revue pourra suggérer des solutions permettant d'éviter cet écueil de l'empilement, d'abord en fournissant des outils conceptuels, ensuite en les enrichissant grâce à des ouvertures sur leurs variantes selon plusieurs aires culturelles, enfin en choisissant des objets de recherche polysémiques propres à être commentés simultanément sous l'angle esthétique - qui me semble très important - qu'historique, social et politique.

\section{MTM :}

Je souhaite que cette revue permette des échanges vivants, qu'elle inspire les jeunes chercheuses et de chercheurs, que les nouvelles générations se sentent encouragées à traiter de thèmes et de notions qui sortent des ornières parfois inhérentes aux frontières disciplinaires et aux anciens schémas de pensée, et pour qu'elles découvrent l'enrichissement et les innovations que les sciences des cultures apportent à la recherche.

\section{$\mathrm{CM}$ :}

J'aimerais que la revue puisse devenir un lieu d'échange et d'expression pour de jeunes chercheurs venant de France, d'Allemagne, mais également d'autres pays, toutes et tous curieux de ce qui se fait ailleurs que dans leur environnement scientifique habituel. Seule un telle ouverture permettra de renouveler et développer des sujets de recherche pertinents et contribuant à un espace franco-allemand et européen de qualité. 